\title{
A CD4 homologue in sea bass (Dicentrarchus labrax): Molecular characterization and structural analysis
}

\author{
Francesco Buonocore ${ }^{\mathrm{a}, *}$, Elisa Randelli ${ }^{a}$, Daniela Casani ${ }^{\mathrm{a}}$, Laura Guerra $^{\mathrm{a}}$, Simona Picchietti $^{\mathrm{a}}$, \\ Susan Costantini ${ }^{\mathrm{b}}$, Angelo M. Facchiano ${ }^{\mathrm{b}}$, Jun Zou ${ }^{\mathrm{c}}$, Chris J. Secombes ${ }^{\mathrm{c}}$, Giuseppe Scapigliati ${ }^{\mathrm{a}}$ \\ a Dipartimento di Scienze Ambientali, University of Tuscia, Largo dell'Università s.n.c., 01100 Viterbo, Italy \\ ${ }^{\mathrm{b}}$ Laboratorio di Bioinformatica e Biologia Computazionale, Istituto di Scienze dell'Alimentazione, CNR, I-83100 Avellino, Italy

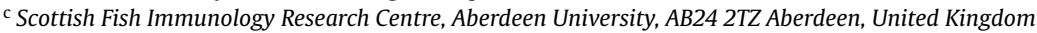

\section{A R T I C L E I N F O}

\section{Article history:}

Received 20 December 2007

Accepted 29 February 2008

Available online 9 April 2008

\section{Keywords:}

CD4

Dicentrarchus labrax

Real-time PCR

3D structure

MHC class II

\begin{abstract}
A B S T R A C T
CD4 is a transmembrane glycoprotein fundamental for cell-mediated immunity. Its action as a T cell coreceptor increases the avidity of association between a T cell and an antigen-presenting cell by interacting with portions of the complex between MHC class II and TR molecules. In this paper we report the cDNA cloning, expression and structural analysis of a CD4 homologue from sea bass (Dicentrarchus labrax). The sea bass CD4 cDNA consists of 2071 bp that translates in one reading frame to give the entire molecule containing 480 amino acids. The analysis of the sequence shows the presence of four putative Ig-like domains and that some fundamental structural features, like a disulphide bond in domain D2 and the CXC signalling motif in the cytoplasmic tail, are conserved from sea bass to mammals. Real-time PCR analysis showed that very high levels of CD4 mRNA transcripts are present in thymus, followed by gut and gills. In vitro stimulation of head kidney leukocytes with LPS and PHA-L gave an increase of CD4 mRNA levels after $4 \mathrm{~h}$ and a decrease after $24 \mathrm{~h}$. Homology modelling has been applied to create a 3D model of sea bass CD4 and to investigate its interaction with sea bass MHC-II. The analysis of the 3D complex between sea bass CD4 and sea bass MHC-II suggests that the absence of a disulfide bond in the CD4 D1 domain could make this molecule more flexible, inducing a different conformation and affecting the binding and the way of interaction between CD4 and MHC-II. Our results will add new insights into the sea bass T cell immune responses and will help in the identification of $\mathrm{T}$ cell subsets in teleost fishes to better understand the evolution of cell-mediated immunity from fish to mammals.
\end{abstract}

(c) 2008 Elsevier Ltd. All rights reserved.

\section{Introduction}

The two major subclasses of $\mathrm{T}$ cells, helper $\mathrm{T}\left(\mathrm{T}_{\mathrm{H}}\right)$ cells and cytotoxic $\mathrm{T}\left(\mathrm{T}_{\mathrm{C}}\right)$ cells, are characterized in mammals by different responses to antigens. $\mathrm{T}_{\mathrm{H}}$ cells stimulate the expression and secretion of cytokines that produce antibody responses or lead to macrophage activation, while $\mathrm{T}_{\mathrm{C}}$ cells are involved in the killing of the antigen-bearing cells. The $T$ cell antigen receptor (TR) recognizes a complex formed by a peptide antigen bound to the major histocompatibility complex (MHC) molecules (Wange and Samelson, 1996). The antigens are presented by different types of MHC molecules: antigens presented by class II MHC molecules generally elicit a $T_{H}$ response, whereas antigens presented by class I MHC molecule give rise to a $T_{C}$ response. $C D 8$ and $C D 4$ are $T$ cell co-receptor glycoproteins that participate in antigen recognition

\footnotetext{
* Corresponding author. Tel.: +39 0761 357644; fax: +39 0761357179. E-mail address: fbuono@unitus.it (F. Buonocore).
}

through interactions with non-polymorphic regions of class I and class II MHC molecules, respectively (Salter et al., 1990; Konig et al., 1992; Cammarota et al., 1992). Therefore, CD4-expressing cells are predominantly of the helper phenotype, while CD8-expressing cells of the cytotoxic phenotype (Germain, 2002).

In mammals, CD4 is a transmembrane molecule, belonging to the immunoglobulin superfamily (IgSF), that contains four Ig-like domains (D1-D4), two of which are V-like domains (D1 and D3) and the other two C-like domains (D2 and D4). Moreover, CD4 has a cytoplasmic tail that associates with a tyrosine protein kinase, p56 $6^{\text {lck }}$, that provides the first signal for T cell activation (Lin et al., 1998). Producing diffraction-quality crystals of the entire extracellular region of CD4 proved difficult. However, proteolytic analyses showed that this molecule could be cleaved into stable fragments that proved much more amenable to crystallographic studies. Both TR-independent binding assays and T cell functional assays, in conjunction with mutagenesis of human or murine CD4 molecules, suggested that an extended region on the CD4 D1 and D2 domains interact with class II MHC molecule (Konig et al., 1996). 